\title{
Double-illumination photoacoustic microscopy of intestinal hemodynamics following massive small bowel resection
}

Junjie Yao, Kathryn J. Rowland, Lidai Wang, Konstantin I. Maslov, Brad W. Warner, et al.

Junjie Yao, Kathryn J. Rowland, Lidai Wang, Konstantin I. Maslov, Brad W. Warner, Lihong V. Wang, "Double-illumination photoacoustic microscopy of intestinal hemodynamics following massive small bowel resection," Proc. SPIE 8223, Photons Plus Ultrasound: Imaging and Sensing 2012, 82233V (23 February 2012); doi: 10.1117/12.909508

SPIE. Event: SPIE BiOS, 2012, San Francisco, California, United States 


\title{
Double-illumination Photoacoustic Microscopy of Intestinal Hemodynamics Following Massive Small Bowel Resection
}

\author{
Junjie Yao $^{1 \uparrow}$, Kathryn J. Rowland ${ }^{2 \uparrow}$, Lidai Wang ${ }^{1 \uparrow}$, Konstantin I. Maslov ${ }^{1}$, \\ Brad W. Warner ${ }^{2^{*}}$, Lihong V. Wang ${ }^{1 *}$, \\ ${ }^{1}$ Department of Biomedical Engineering, Washington University in St. Louis, St. Louis, MO \\ 63130, USA \\ ${ }^{2}$ Division of Pediatric Surgery, St. Louis Children's Hospital, Washington University School of \\ Medicine, St. Louis, MO 63110, USA \\ "These authors contributed equally to this work. \\ *Corresponding authors:
}

Brad W. Warner for small bowel resection, brad.warner@wustl.edu

Lihong V. Wang for photoacoustic microscopy, 1hwang@biomed.wustl.edu

\begin{abstract}
Massive small bowel resection (SBR) results in villus angiogenesis and intestinal adaptation. The exact mechanism that causes intestinal villus angiogenesis remains unknown. We hypothesize that hemodynamic changes within the remnant bowel after SBR will trigger intestinal angiogenesis. To validate this, we used photoacoustic microscopy (PAM) to image the microvascular system of the intestine in C57B6 mice and to measure blood flow and oxygen saturation $\left(\mathrm{sO}_{2}\right)$ of a supplying artery and vein. Baseline measurements were made $6 \mathrm{~cm}$ proximal to the ileal-cecal junction (ICJ) prior to resection. A 50\% proximal bowel resection was then performed, and measurements were again recorded at the same location immediately, 1,3 and 7 days following resection. The results show that arterial and venous $\mathrm{SO}_{2}$ were similar prior to SBR. Immediately following SBR, the arterial and venous $\mathrm{sO}_{2}$ decreased by $14.3 \pm 2.7 \%$ and $32.7 \pm 6.6 \%$, respectively, while the arterial and venous flow speed decreased by $62.9 \pm 17.3 \%$ and $60.0 \pm 20.1 \%$, respectively. Such significant decreases in $\mathrm{sO}_{2}$ and blood flow indicate a hypoxic state after SBR. Within one week after SBR, both $\mathrm{sO}_{2}$ and blood flow speed had gradually recovered. By 7 days after SBR, arterial and venous $\mathrm{sO}_{2}$ had increased to $101.0 \pm 2.9 \%$ and $82.7 \pm 7.3 \%$ of the baseline values, respectively, while arterial and venous flow speed had increased to $106.0 \pm 21.4 \%$ and $150.0 \pm 29.6 \%$ of the baseline values, respectively. Such increases in $\mathrm{sO}_{2}$ and blood flow may result from angiogenesis following $\mathrm{SBR}$.
\end{abstract}

Keywords: photoacoustic microscopy, small bowel resection, oxygen saturation, blood flow, hypoxia, angiogenesis

\section{Introduction}

Short bowel syndrome (SBS), a malabsorption disorder usually caused by the surgical removal of the small intestine, is a high-risk condition within the pediatric patients. After a massive small bowel resection (SBR) in both animal models and humans, a critical adaptation response occurs within the remnant bowel, including increased villus height and crypt depth, and thus increased absorptive mucosal surface area, to compensate for the attenuated bowel length [1-3]. As angiogenesis has long been observed as an important factor for cellular proliferation [4, 5], it has also been demonstrated that villus angiogenesis contributes to intestinal adaptation after SBR [6-9]. However, the exact stimulus for these proangiogenic changes as well as the acute alterations in intestinal hemodynamics following SBR are presently unknown.

Photons Plus Ultrasound: Imaging and Sensing 2012, edited by Alexander A. Oraevsky, Lihong V. Wang, Proc. of SPIE Vol. 8223, 82233V · C 2012 SPIE · CCC code: 1605-7422/12/\$18 - doi: 10.1117/12.909508 
Previous ex vivo studies of intestinal blood flow at late time points after SBR are not consistent and insufficient [1012]. An in vivo, real-time imaging modality with endogenous contrast is therefore needed to measure the hemodynamic parameters within the remnant bowel both immediately and several days after bowel resection, including blood flow speed, oxygen saturation of hemoglobin $\left(\mathrm{sO}_{2}\right)$ and metabolic rate of oxygen $\left(\mathrm{MRO}_{2}\right)$. Photoacoustic microscopy (PAM), a non-invasive, label-free, high-resolution hybrid imaging modality, is a promising tool for such measurements. PAM takes advantage of both rich optical absorption contrast and weak ultrasonic scattering in biomedical tissue, and thus yields high-contrast images with relatively deep penetration [13]. By spectrally unmixing contributions from various endogenous or exogenous chromophores, PAM is capable of anatomical, functional, molecular, flow dynamic and metabolic imaging [13-24]. With the help of PAM, a better understanding of the acute hemodynamic changes following SBR may further elucidate a mechanism for villus angiogenesis and the pathogenesis of intestinal adaptation.

\section{Materials and methods}

\section{Experimental design}

A protocol for this study was approved by the Washington University Animal Studies Committee (20090275) in accordance with the National Institute of Health laboratory animal care and use guidelines. Male mice (C57BL/6; Harlan Laboratories, Inc.; Indianapolis, IN) age 8 to 15 weeks were used in this study. Mice underwent either 50\% proximal SBR or sham (enterotomy alone) as previously reported [1]. Briefly, a midline laparotomy was made and the bowel was exposed for photoacoustic measurements immediately, 1, 3 and 7 days after the operation. Mice that underwent SBR had transection of the bowel at $6 \mathrm{~cm}$ proximal to the ICJ and at 1 to $2 \mathrm{~cm}$ distal to the ligament of Treitz. The mesentery of the intervening bowel was ligated with a silk tie and the intervening bowel was removed. In mice that underwent the sham procedure, the bowel was transected only at $6 \mathrm{~cm}$ proximal to the ICJ.

\section{Double-illumination photoacoustic microscopy of intestinal $\mathrm{s}_{2}$ and blood flow}

A newly developed double-illumination photoacoustic microscopy (DI-PAM) was used for this study in order to see through the whole cross-section of the small bowel [25] (Fig. 1). The short laser pulses are reshaped by an iris (ID25SS, Thorlabs) and attenuated by a neutral density filter (NDC-50C-2M, Thorlabs). The attenuated beam is then split into two sub-beams (top and bottom) by a 50/50 beam splitter (BSW04, Thorlabs). The top beam is focused by a condenser lens (LA1131, Thorlabs) before passing through a $25 \mu \mathrm{m}$ pinhole (P50C, Thorlabs) for further spatial filtering. The filtered beam is then focused by an optical objective (AC127-050-A, Thorlabs. NA: 0.1 in air) into the sample from the top. A beam combiner composed of a thin layer of silicone oil sandwiched by a right-angle prism (NT32-545, EdmundOptics) and a rhomboid prism (NT49-419, EdmundOptics) provides acoustic-optical coaxial alignment. The resultant photoacoustic waves are detected by an ultrasonic transducer (V214-BB-RM, OlympusNDT) with a central frequency of $50 \mathrm{MHz}$. An acoustic lens with an NA of 0.5 is ground into the bottom of the rhomboid prism to provide an acoustic focal diameter of $30 \mu \mathrm{m}$. An optical correction lens is attached to the top of the beam combiner to correct the aberration of the glass prisms. The acoustic lens is submerged in a water-tank for ultrasound coupling. The bottom beam duplicates the same path as the top beam, except that it is directly focused into the sample from the bottom without going through the beam combiner and water tank. By carefully adjusting the positions of two objectives, we achieved a co-axial configuration of the two optical foci and the acoustic focus. To best expand the optical focal zone, the top and bottom optical foci are both approximately one Rayleigh range apart from the acoustic focus. 


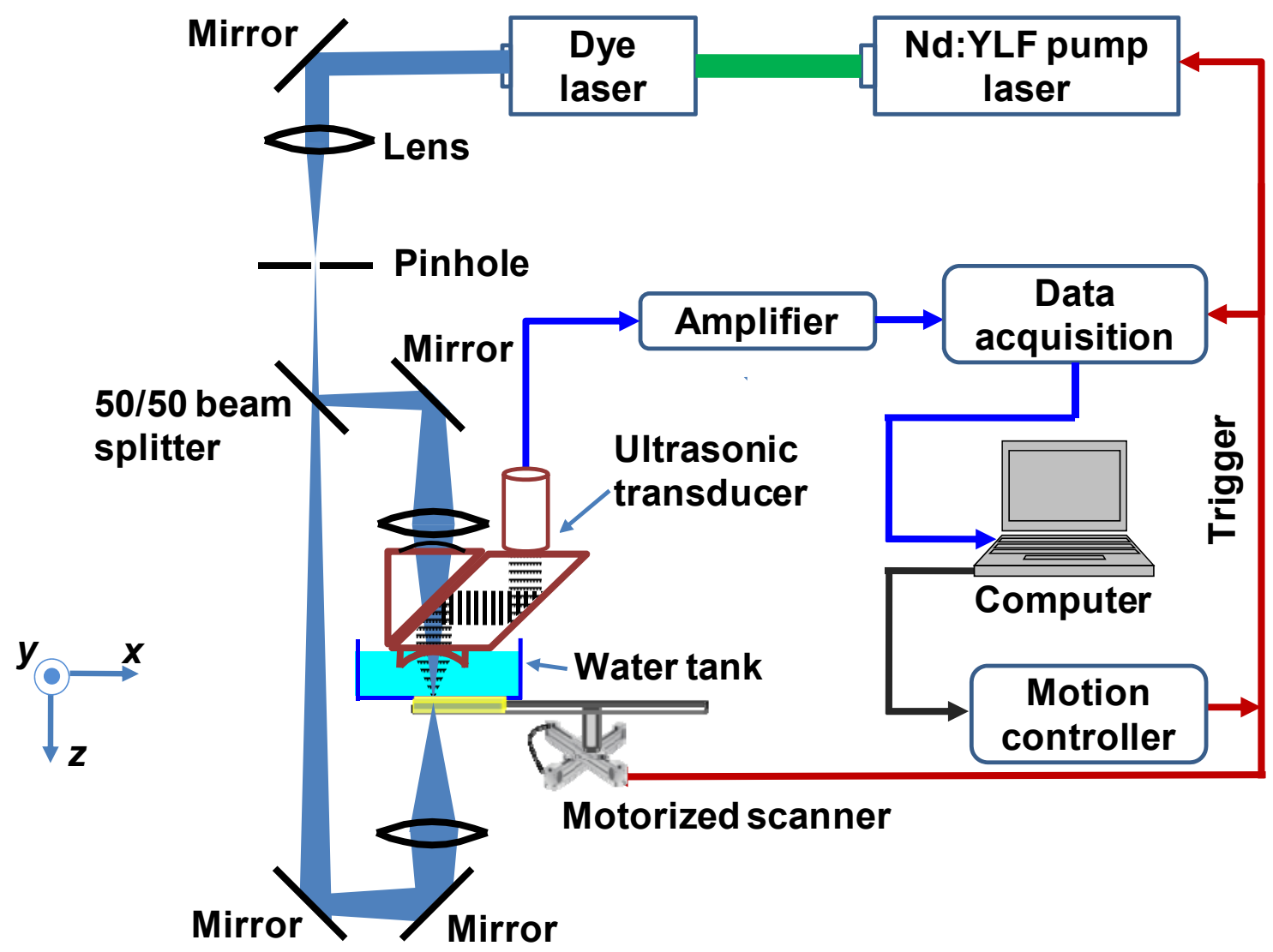

Figure 1. System schematic of double-illumination photoacoustic microscopy.

During the experiment, mice were anesthetized with isoflurane (E-Z Anesthesia, Euthanex) and placed in a supine position on a heating pad $\left(37^{\circ} \mathrm{C}\right)$. A midline laparotomy was performed and the small bowel was exposed. The terminal mesenteric artery and accompanying vein at a point approximately $6 \mathrm{~cm}$ proximal to the ICJ were identified. Baseline $\mathrm{sO}_{2}$ was measured at both locations on a $1 \times 4 \mathrm{~mm}^{2}$ area containing such vessel pairs at two optical wavelengths of $570 \mathrm{~nm}$ and $578 \mathrm{~nm}$ using a previous publish method [21]. Baseline blood flow speed measurement was then performed at both locations across the proximal end of the vessel in $M$-mode using a bandwidth broadening based method $[19,20]$. The laser repetition rate was $8 \mathrm{kHz}$, and $3200 \mathrm{~A}$-lines were acquired at each position. The area of the bowel not being measured was kept moistened with a normal saline soaked gauze pad. The animal then underwent SBR or sham procedure. Following the procedure, the same artery and vein pairs at $6 \mathrm{~cm}$ proximal to the ICJ were imaged with $\mathrm{sO}_{2}$ and blood flow measurements recorded. For the animals imaged 1,3 and 7 days after the SBR or sham, there were no baseline measurements performed. Following all measurements the animal was sacrificed via cervical dislocation. For each time point, a total of 7 mice underwent the SBR procedure and 7 mice underwent the sham procedure. All the photoacoustic data processing was conducted using MATLAB (R2008a, MathWorks). Quantitative values are presented as mean \pm SEM.

\section{Results}

\section{Double-illumination PAM of small intestine}

With the help of the extended imaging depth, the whole cross-section of the intestine was successfully imaged by DI-PAM (Fig. 2) [25]. The results show that the dense vessels on the intestine wall would block the excitation light penetrating through the top half [Fig. 2(A)] or bottom half [Fig. 2(B)] of the intestine under top or bottom illumination, respectively. Only the double illumination provided the vasculature information from both halves [Fig. $2(\mathrm{C})]$. 
(A)

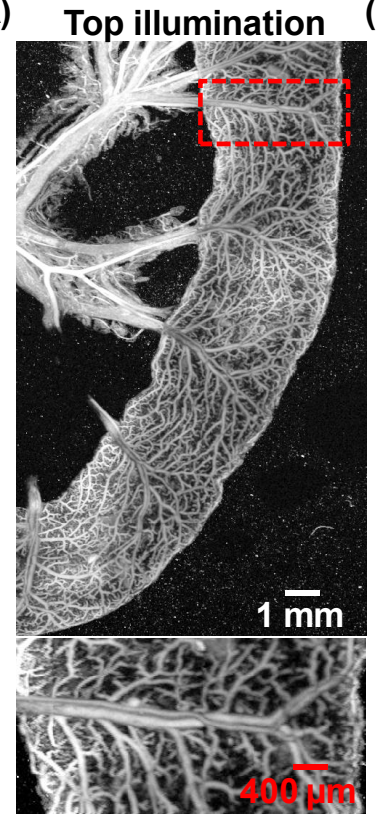

(B)

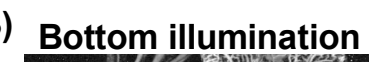

(C)

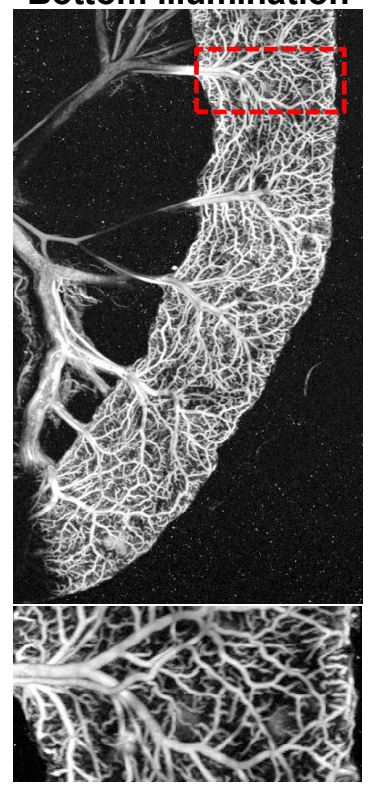

Double illumination

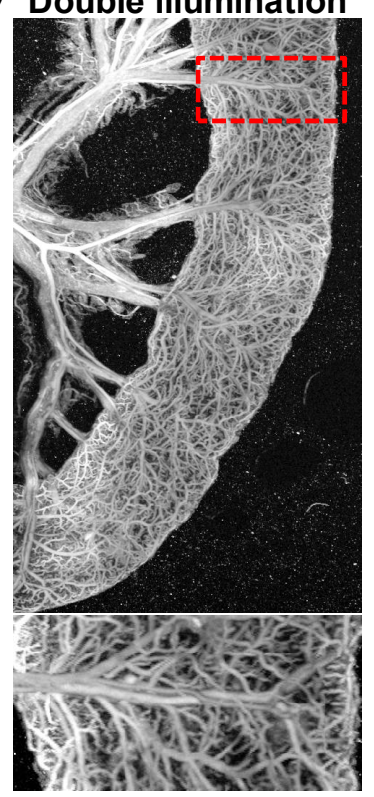

Figure 2. PAM of small bowel intestine under (A) top illumination, (B) bottom illumination, (C) double-illumination, respectively. The bottom row is the close-up of the dashed box in the top row.

\section{Arterial and venous oxygen saturation}

Prior to intervention, arterial and venous $\mathrm{sO}_{2}$ of the terminal mesenteric artery and accompanying vein were similar. This may indicate minimal to no tissue oxygen utilization of the supplied intestine, which suggests either a high physiologic reserve in mice, and/or left-to-right shunting within the intestinal wall even with a metabolically active state.

Immediately following SBR, the arterial oxygen saturation decreased (0.98 \pm 0.01 pre vs. $0.84 \pm 0.06$ post-SBR). This decrease in arterial oxygen saturation was also observed following sham $(0.98 \pm 0.01$ pre vs. $0.86 \pm 0.04$ postsham) [Fig. 3(A)]. Venous oxygen saturation dramatically decreased immediately following SBR $(0.98 \pm 0.02$ pre vs $0.66 \pm 0.05$ post-SBR) [Fig. 3(B)]. This decrease in venous oxygen saturation was also observed to a lesser degree following sham $\left(0.98 \pm 0.01\right.$ pre vs $0.86 \pm 0.02$ post-sham). By one week after $\mathrm{SBR}$, arterial and venous $\mathrm{sO}_{2}$ had increased to $101.0 \pm 2.9 \%$ and $82.7 \pm 7.3 \%$ of the baseline values, respectively. By one week after sham, arterial and venous $\mathrm{sO}_{2}$ had increased to $99.0 \pm 5.7 \%$ and $102.1 \pm 3.2 \%$ of the baseline values, respectively [Fig. 3(A-B)] 
(A)

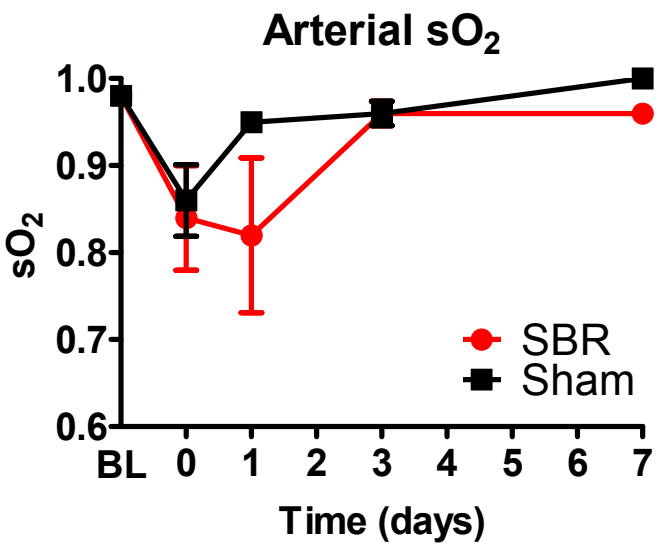

(B)

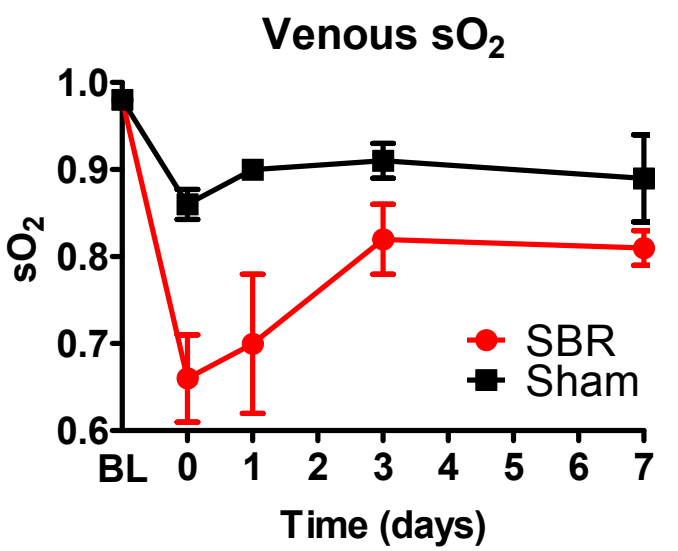

Figure 3. The measured oxygen saturation of the artey (A) and vein (B) before and after SBR or sham operations.

\section{Arterial and venous blood flow}

Immediately following SBR, the arterial blood flow speed decreased $(7.6 \pm 1.5 \mathrm{~mm} / \mathrm{s}$ pre vs. $2.6 \pm 0.50 \mathrm{~mm} / \mathrm{s}$ postSBR). This decrease in arterial flow speed was not observed following sham $(7.6 \pm 1.5 \mathrm{~mm} / \mathrm{s}$ pre vs. $7.7 \pm 0.60$ $\mathrm{mm} / \mathrm{s}$ post-sham) [Fig. 4(A)]. Venous flow speed decreased immediately following SBR $(4.0 \pm 0.70 \mathrm{~mm} / \mathrm{s}$ pre vs 1.6 $\pm 0.50 \mathrm{~mm} / \mathrm{s} \mathrm{post-SBR})$. This decrease in venous flow speed was not observed following sham $(4.0 \pm 0.70 \mathrm{~mm} / \mathrm{s}$ pre vs $4.0 \pm 0.45 \mathrm{~mm} / \mathrm{s}$ post-sham) [Fig. 4(B)]. By one week after SBR, arterial and venous flow speed had increased to $106.0 \pm 21.4 \%$ and $150.0 \pm 29.6 \%$ of the baseline values, respectively. By one week after sham, arterial and venous flow speed returned to the baseline values [Figs. 4(A-B)].

(A)

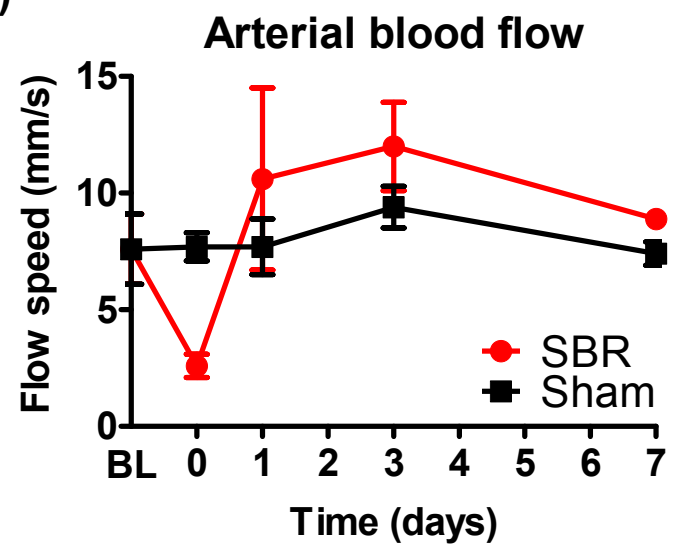

(B)

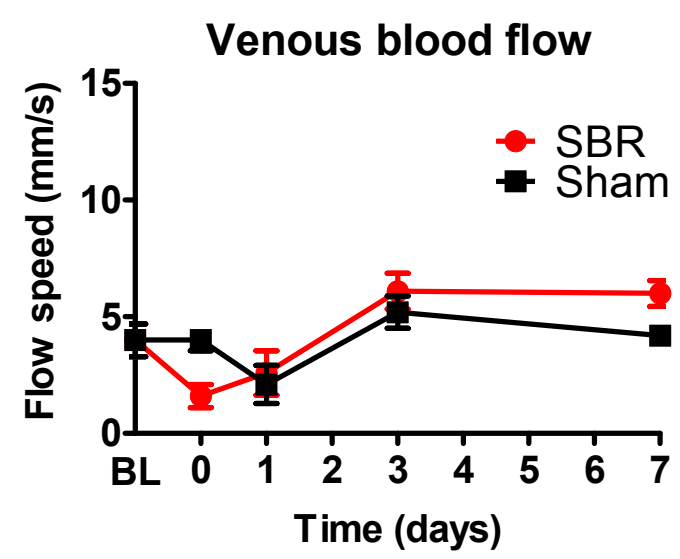

Figure 4. The measured blood flow speed of the artey (A) and vein (B) before and after SBR or sham operations.

\section{Conclusion and discussion}

Immediately following SBR, hemodynamic changes occur consistent with a reduced oxygen delivery. Venous $\mathrm{sO}_{2}$ drops dramatically post-SBR. A less dramatic decrease in venous $\mathrm{sO}_{2}$ also occurs post-sham, likely related to the metabolic effects of transection alone. Further, both arterial and venous blood flow decreased post-SBR. Such decrease in blood flow was not seen post-sham. The dramatic decrease of $\mathrm{sO}_{2}$ and blood flow indicates a hypoxia environment immediately following SBR. Hypoxia is a well-recognized trigger of angiogenesis, resulting in the activation of hypoxia-inducible factors (HIF), responsible for transcriptional activation of genes. 
Seven days following the $\mathrm{SBR}$, while the arterial $\mathrm{sO}_{2}$ had recovered to the baseline, the venous $\mathrm{sO}_{2}$ was still lower than the baseline. This indicates an increase in the oxygen extraction fraction. In combining with the significantly increased blood flow speed, it is safe to conclude that the metabolic rate of oxygen $\left(\mathrm{MRO}_{2}\right)$ of the remnant bowel was increased [15]. Such increase suggested a hypermetabolism induced by massive SBR. In contrast, there is no increase in the $\mathrm{MRO}_{2}$ following sham.

The present study demonstrates that PAM is a useful tool for measuring intestinal hemodynamics. The use of hemoglobin as endogenous contrast eliminates the potential disturbance to the intestinal system induced by exogenous contrast. In addition, the minimum invasiveness of PAM enables longitudinal studies on the same animal. Through the use of photoacoustic microscopy, the duration and cellular effects of intestinal hypoxia and villus angiogenesis can be further studied, which may help in the development of future therapeutic treatments for patients with short bowel syndrome.

\section{Acknowledgements}

Wang laboratory research was supported by the National Institutes of Health Grants R01 EB000712, R01 EB008085, R01 CA134539, U54 CA136398, R01 EB010049, R01 CA157277, and 5P60 DK02057933. L.V.W. has a financial interest in Microphotoacoustics, Inc. and Endra, Inc., which, however, did not support this work.

Warner laboratory research was support by National Institutes of Health Grants R01 DK059288 (Warner) and T32 CA009621 (Rowland).

The authors thank Yan Liu and Joon-Mo Yang for useful discussion and technical assistance.

\section{References}

[1] Helmrath, M. A., et al., "Intestinal adaptation following massive small bowel resection in the mouse.," Journal of the American College of Surgeons. 183(5), 441-449 (1996).

[2] Taylor, J. A., et al., "Lessons learned: optimization of a murine small bowel resection model," Journal of Pediatric Surgery. 43(6), 1018-1024 (2008).

[3] McDuffie, L. A., et al., "Intestinal adaptation after small bowel resection in human infants," Journal of Pediatric Surgery. 46(6), 1045-1051 (2011).

[4] Folkman, J., "Is angiogenesis an organizing principle in biology and medicine?," Journal of Pediatric Surgery. 42(1), 1-11 (2007).

[5] Folkman, J., et al., "Tumor Angiogenesis - Therapeutic Implications," New England Journal of Medicine. 285(21), 1182-\& (1971).

[6] Lee, K. D., et al., "Basic fibroblast growth factor and granulocyte colony-stimulating factor enhance mucosal surface expansion after adult small bowel transplantation without vascular reconstruction in rats," Journal of Pediatric Surgery. 41(4), 737-741 (2006).

[7] Parvadia, J. K., et al., "Role of VEGF in small bowel adaptation after resection: the adaptive response is angiogenesis dependent," American Journal of Physiology-Gastrointestinal and Liver Physiology. 293(3), G591-G598 (2007).

[8] Martin, C. A., et al., "Intestinal resection induces angiogenesis within adapting intestinal villi," Journal of Pediatric Surgery. 44(6), 1077-1083 (2009).

[9] McMellen, M. E., et al., "Epidermal growth factor receptor signaling modulates chemokine (CXC) ligand 5 expression and is associated with villus angiogenesis after small bowel resection," Surgery. 148(2), 364-70 (2010).

[10] Ulrich-Baker, M. G., et al., "Blood flow responses to small bowel resection," Am J Physiol. 251(6 Pt 1), G815-22 (1986).

[11] Touloukian, R. J. and Spencer, R. P., "Ileal blood flow preceding compensatory intestinal hypertrophy," Ann Surg. 175(3), 320-5 (1972).

[12] Jesseph, J. E. and Jacklin, A. J., "Effects of Partial Resection of Mammalian Small Intestine. Iii. Glucose Absorption, Co2 Production and Blood Flow in Residual Ileum in the Dog," Rev Surg. 20, 384-7 (1963). 
[13] Kim, C., Favazza, C., and Wang, L. H. V., "In Vivo Photoacoustic Tomography of Chemicals: HighResolution Functional and Molecular Optical Imaging at New Depths," Chemical Reviews. 110(5), 2756$2782(2010)$.

[14] Yao, J., et al., "Evans blue dye-enhanced capillary-resolution photoacoustic microscopy in vivo," Journal of Biomedical Optics. 14(5), 054049 (2009).

[15] Yao, J., et al., "Label-free oxygen-metabolic photoacoustic microscopy in vivo," Journal of Biomedical Optics. 16(7), 076003 (2011).

[16] Maslov, K., et al., "Optical-resolution photoacoustic microscopy for in vivo imaging of single capillaries," Opt Lett. 33(9), 929-31 (2008).

[17] Wang, L., et al., "Fast voice-coil scanning optical-resolution photoacoustic microscopy," Opt Lett. 36(2), 139-41 (2011).

[18] Wang, X., et al., "Noninvasive laser-induced photoacoustic tomography for structural and functional in vivo imaging of the brain," Nat Biotechnol. 21(7), 803-6 (2003).

[19] Yao, J., et al., "In vivo photoacoustic imaging of transverse blood flow by using Doppler broadening of bandwidth," Opt. Lett. 35(9), 1419-1421 (2010).

[20] Yao, J. and Wang, L. V., "Transverse flow imaging based on photoacoustic Doppler bandwidth broadening," Journal of Biomedical Optics. 15(2), 021304 (2010).

[21] Zhang, H. F., et al., "Imaging of hemoglobin oxygen saturation variations in single vessels in vivo using photoacoustic microscopy," Applied Physics Letters. 90(5), 053901 (2007).

[22] Zhang, H. F., et al., "Functional photoacoustic microscopy for high-resolution and noninvasive in vivo imaging," Nat Biotechnol. 24(7), 848-51 (2006).

[23] Yao, J., et al., "Label-free oxygen-metabolic photoacoustic microscopy in vivo," Journal of Biomedical Optics. 16(7), 076003-11 (2011).

[24] Zhang, C., Maslov, K., and Wang, L. V., "Subwavelength-resolution label-free photoacoustic microscopy of optical absorption in vivo," Opt Lett. 35(19), 3195-7 (2010).

[25] Yao, J., et al., "Double-illumination photoacoustic microscopy," Opt. Lett. 37(5), in press (2012). 